\title{
Challenging the Use of Bandage Compression as the Baseline for Evaluating the Healing Outcomes of Venous Leg Ulcer-Related Compression Therapies in the Community and Outpatient Setting: An Integrative Review
}

\author{
Maryam Asaf ${ }^{a}$ Nezar Salim $^{b}$ Mohammed Tuffaha ${ }^{c}$ \\ a University of Sydney, Sydney, NSW, Australia; ${ }^{\text {b}}$ Education Department, Dubai Hospital, Dubai Health Authority, \\ Dubai, UAE; ' Amana Health Care Medical and Rehabilitation Hospital, Abu Dhabi, UAE
}

\section{Keywords}

Bandage compression · Venous leg ulcer · Compression therapies

\begin{abstract}
Background: Venous ulcers have considerable human and financial costs. Bandage compression is the most common type of compression used to enhance healing. Ulcer size, pretreatment ulcer duration, and the consistency of pressure (depending on the competency of the bandager) significantly affect the healing process. Summary: The aim of this review is to explore whether the variability in bandagerelated baselines contributes to the differences in healing outcomes among the empirical studies designed to evaluate bandage compression for treating venous ulcers in community and outpatient settings. Two health-related databases were searched: Medline and the Cumulative Index for Nursing and Allied Health. A manual search of relevant reviews and publication reference lists was also undertaken. Fourteen studies were included. The most common study design was a randomized controlled trial. Only one study used a cohort design. This review focused on two variables: healing
\end{abstract}

rate and healing time. The ulcer features, the consistency of the pressure level, and the competency of the bandager were the main factors within the reviewed studies that affect the baselines and healing outcomes. Key Messages: This integrative review confirms that the large variability in the baselines results from differences in the ulcer size, duration, competency level, and amount of pressure that led to differing healing outcomes. Future research designs need to pay attention to make the baselines consistent by ensuring that the ulcer size, duration, competency of the nurses, and level of compression are comparable and consistent within the studies' design.

(C) 2018 The Author(s)

Published by S. Karger AG, Basel

\section{Introduction}

Venous leg ulcers (VLUs), defined as open skin lesions on the foot or leg, mostly occur between the ankles and the knees as a consequence of venous hypertension [1]. VLUs are the most common etiology of leg ulcers among the adult population. Globally, the prevalence rate of VLUs is $1 \%$ of the adults aged $18-64$ years. About $5 \%$ of 
the adults $>65$ years of age suffer from venous ulcers, and this number continues to rise due to the global ageing population $[2,3]$.

VLUs typically present in a repeated cycle of ulceration, healing, and recurrence. VLUs can take months or years to heal or also remain unhealed $[4,5]$. The 12-month recurrence rates between 18 and $28 \%[6,7]$. VLUs are the most common type of leg ulcer and accounting for almost $80 \%$ of all types of leg ulcers $[1,5,8]$. Significantly, up to $80 \%$ of the treatment and application of compressions for VLUs are performed in community and outpatient settings [9-11].

Globally, VLUs present a tremendous cost burden for both patients and the healthcare system. However, the cost for treatment varies in different countries. For example, in the United Kingdom, the estimated annual cost of VLU treatment is GBP 941.1 million to treat approximately 300,000 patients [12]. In Australia, the annual cost of VLU treatment is estimated to be USD 784.66 million to treat 47,299 cases. In the United States, approximately USD 2.5 billion was spent to treat 6 million patients with VLU [13-15]. In the United Kingdom, Vowden and Vowden [16] estimated that GBP 1.69 million was spent on compression therapy and wound dressing, while a further GBP 3.08 million was spent on nurses' salaries who then spent more than $50 \%$ of their working time treating VLUs through compression therapy.

Bandage compression is the most common type of compression therapy when treating VLUs and it is routinely applied to aid venous hypertension and promote healing to increase the healing rate (HR) and shorten the healing time (HT). The three most common types of bandage compression have been known for centuries [17].

Healing or closure of a VLU is defined as complete epithelialization without drainage or the need for a dressing over the closed ulceration, although venous compression would still be required $[18,19]$. The average HT is 113 days (range 90-135) [20]. Healing outcomes are affected by many factors, which lead either to the positive promotion of healing outcomes or the negative delay of healing outcomes [21].

Bandaging was the first approach used to provide compression to treat VLUs. However, the consistent pressure of bandaging has been a long-term problem. Healing outcomes were found to vary widely ( $\mathrm{HT}=40$ 138 days; $H R=51-93 \%)[18,22,23]$. Studies have been unable to demonstrate the superiority of multi-layer bandage compression over other types [24, 25]. Therefore, previous findings of differences in healing outcomes have raised questions about whether the inconsistent baselines in the studies contributed to the variations in the healing outcomes.

This integrative review aims to explore whether the variability in the bandage-related baselines contributes to the differences in healing outcomes among the empirical studies designed to evaluate the effect of bandage compression for treating VLU in community and outpatient settings.

\section{Methodology}

The search was conducted using MEDLINE and Cumulative Index to Nursing and Allied Health Literature (CINAHL). The primary search terms were "compression therapy" and "venous leg ulcer." The search was restricted to peer-reviewed primary and empirical studies published in English in the field of human studies. The search was limited to studies published between 2002 and 2017 because quantitative studies prior to 2002 lacked methodological rigor and statistical precision, making it impossible to provide precise measurements of the effect of an intervention on a specific population [26]. A manual search of relevant reviews and publication reference lists was also conducted to reduce the risk of missing relevant papers.

The electronic and manual searches identified a total of 1,739 citations as of August 20, 2017. The citations were imported to EndNote X ${ }^{\circledR}$ (Thomson Reuters, New York, NY, USA). After removing 355 duplicate articles, the abstracts and titles of 1,384 articles were assessed for eligibility against the inclusion criteria. A further 1,335 studies were excluded after screening the abstracts and titles; therefore, 49 articles were reviewed. After the full texts of the 49 articles were reviewed, 35 were excluded, leaving14 articles that met the inclusion criteria and were included in the integrative review.

The remaining 14 peer-reviewed empirical studies were conducted using two types of research design: randomized controlled trial and cohort design. This review focuses on two major variables: HT and HR. Total HT is defined as the time required for the ulcer to heal completely. Table 1 provides an analytical summary of the 14 studies, comparing the different compression therapies.

\section{Results}

This integrative review initially aimed to determine the effectiveness (HR and HT) of bandage compression using stockings, Unna boot, and intermittent pneumatic compression. After relevant studies had been identified, however, data abstraction showed considerable variation in the sample size and reported effectiveness $(n=9-227$; HT 45-112 days; HR 17-85\%). The absence of a reliable foundation of the research rendered further comparison problematic. Accordingly, this review has focused only on variables reported for bandage compression as the ba- 


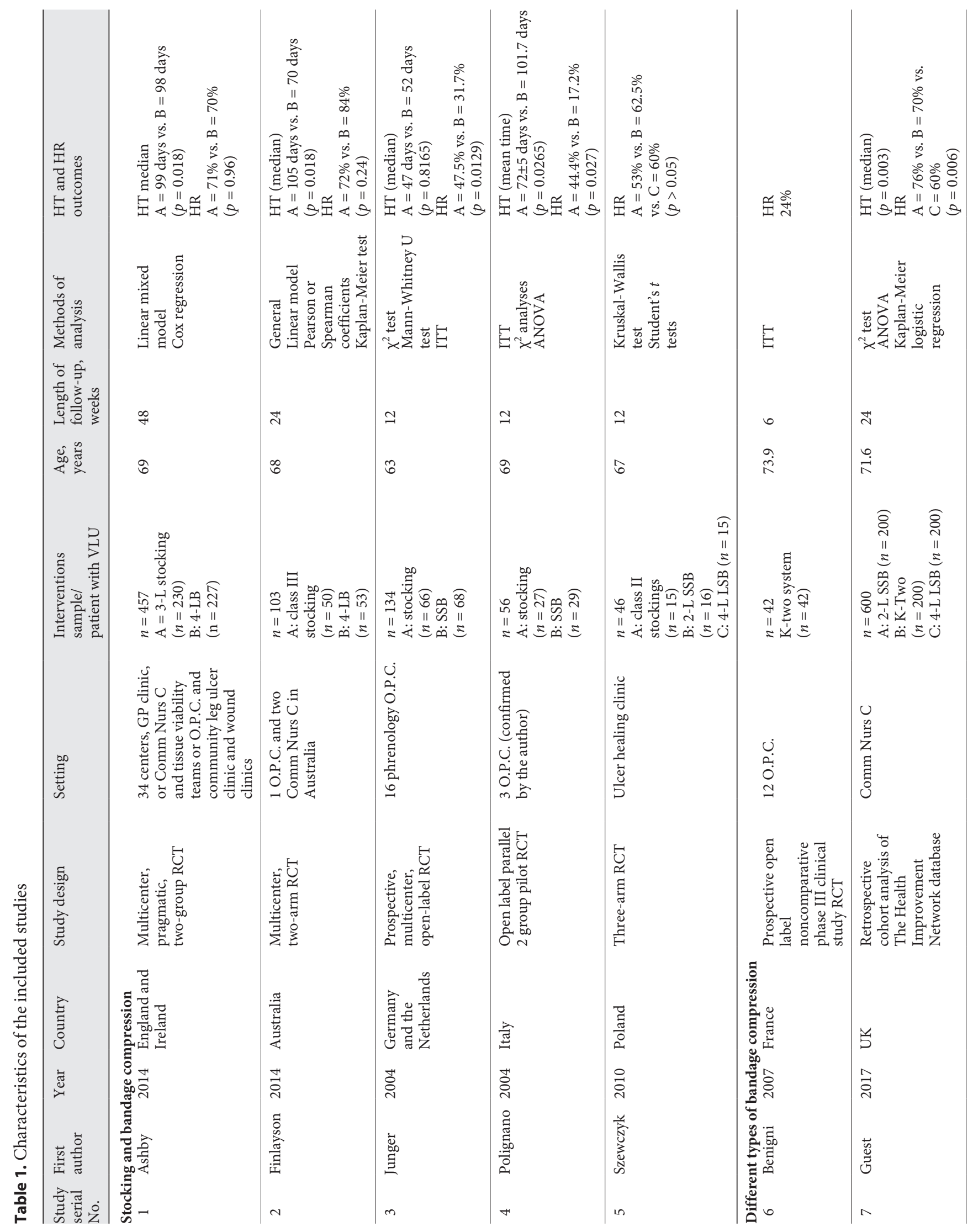




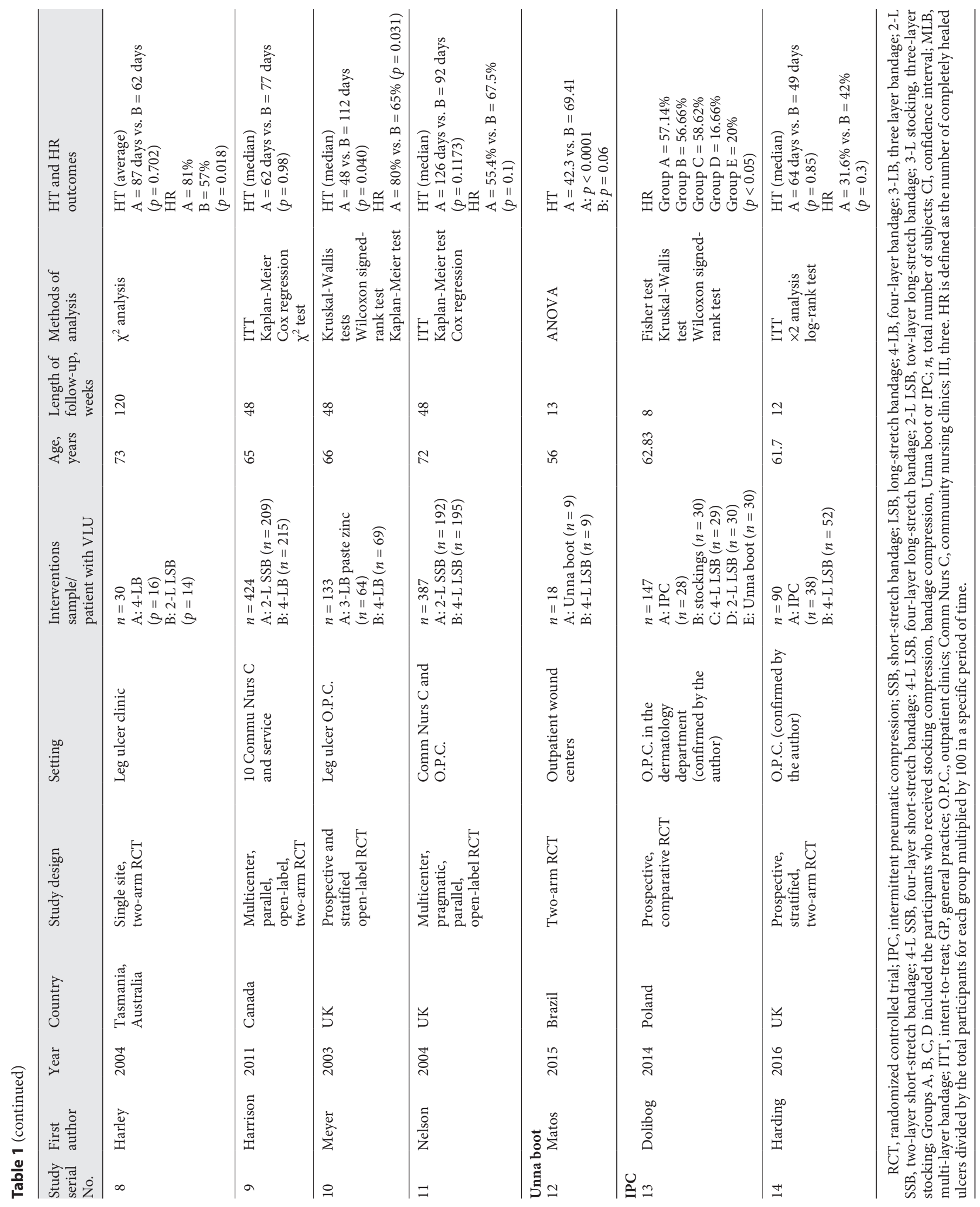


sis for comparisons to determine the causes of the identified variations in HR and HT.

Several factors might have contributed to the variability in the results for both HT and HR: (a) variation in ulcer size, (b) variation in the duration of ulcer pretreatment, and (c) variation in the consistent maintenance of bandaging pressure (compressions), the latter in turn due to varying skill levels among bandage applicators.

\section{Discussion}

\section{Impact on $H T$}

In this review, six types of bandage compression were studied in relation to HT outcome, including four-layer bandage (4-LB), short-stretch bandage (SSB) compression (without defining the number of layers), two-L SSB, four-L SSB, four-L long-stretch bandage (LSB), and twoL LSB. In the analysis of this review, important differences in the size and duration of the ulcers are the main source of the heterogeneity in the baselines, which in turn led to differences in the HT findings among the reviewed studies. Thus, the major limitation in the findings of the reviewed studies is the substantial heterogeneity itself among ulcer features (size and commencement duration). The inclusion of large ulcers with long durations in some of the reviewed studies and the inclusion of small ulcers with short durations in others is the most likely cause of the differences in the HT among the reviewed studies [27-36]. This result is in line with the observational study by Escaleira et al. [38] who included ulcers varying in size from large to small and of durations from short to long and found that this accounted for a significant variance in HTs in the study. However, this study by Escaleira et al. [37] is different from the reviewed studies because the medical dressings were applied before the bandage compression, which can contribute to positive HT findings. Small ulcers with short durations are more likely to heal, and their inclusion in the studies can shorten HT [38].

The variability in the amount of the pressure due to differences in bandager competence is another main factor causing variability in baselines and the HT findings [39-41]. In the studies that review the competency of the applicators, the consistency of the applied pressure was confirmed as one of the main factors that underline the variability in the baselines and HTs of bandage studies of strength in most of the reviewed studies [27-36]. Highly competent applicators applied and maintained the most consistent pressure, which in turn shortened HT [27-31, 33, 34]. Conversely, in two studies, low-skilled applicators applied an inappropriate amount of pressure, which in turn caused a lengthy HT $[32,36]$. It would be useful if future trials could collect data on the staff's skill level at baseline, and this information could be included as a covariate in the modelling of treatment effects.

\section{Impact on $H R$}

Similar issues affected baselines of the reviewed studies in relation to HR. Briefly, several authors reported that differences in the HRs are explained by the vast heterogeneity in the baselines due to the inclusion of ulcers with different sizes and of different duration $[18,19]$. Our results confirm the above reports and show that the inclusion of ulcers of different sizes (large and short) and ulcers of different durations (short and long) contribute to severe heterogeneity in the baselines and differences in the HR among the reviewed bandages of four-LB, SSB compression, two-L SSB, four-L LSB, two-L LSB, and KTwo $[12,24,27-30,32,34,35,42-44]$. Again, this heterogeneity is still a significant limitation in the design of the current research, which needs to be addressed in future clinical studies. Furthermore, the same concern about competency of the bandager and consistent pressure exists for HR.

\section{Implications for Research}

The differences in baselines suggest that future research designs need to pay close attention to ulcer size and to pretreatment duration and consistency in the amount of pressure applied by skilled nurses. It is important that studies include small-sized ulcer sizes and ulcers of short duration and ensure that consistent pressure is applied and maintained. They should also ensure that these factors are similar and comparable among the studies that evaluate the effect of different types of bandages. This will enable meaningful comparisons of the effectiveness of different types of bandages to be made. This is important to build a strong body of evidence in this area and enable patients and health professionals to make informed choices. Future trials should assess the bandage application skills of participating nurses at the baseline to reduce the heterogeneity in skill based on different levels of training. This review highlights the need for future studies to evaluate whether training enhances the competency of the bandager to ensure that all participant bandagers have the same level of skill and apply comparable levels of compression. 


\section{Limitations}

This integrative review has limitations. First, this review was limited to English language studies, which might have eliminated some relevant studies published in other languages. Second, only studies conducted in community and outpatient settings were included in this review. It could be valuable to extend this analysis for the hospital and home care setting.

\section{Conclusion}

This review sought to explore whether the baselines are inconsistent and contribute to discrepancies in HT and HR findings in the studies that examine the bandage compression for treating VLU. This review was limited to studies that used bandage compression as the first approach to provide compression to treat VLUs; scenarios where the application of the consistent pressure has been a long-term problem. Bandage compression is the most common form of compression therapy for treating VLU. This review shows that bandage compression studies reporting variable healing outcomes are the result of severe heterogeneity in baselines in relation to differences ulcer sizes and durations, different bandager experience levels, and the amount of pressure delivered. This variability and inconsistency in baselines limited the feasibility of effec- tively comparing the bandage studies themselves due to insufficient clinical evidence. Therefore, it is difficult to draw a definitive conclusion about the superiority of any specific compression therapy.

As noted above, the major identified gap in the current research is the heterogeneity of the baselines, which in turn affects the HT and HR findings and comparisons of effectiveness in the bandage studies. Thus, future clinical studies need to establish designs that have homogeneous and consistent baselines of clinical evidence. This requires the inclusion of comparable and similar sizes and durations of ulcers, levels of competency, and consistency of pressure. Such study designs should also build on the previous work in order to establish robust clinical evidence that can influence health care decisions regarding VLU treatment.

\section{Disclosure Statement}

The authors have no conflicts of interest to declare.

\section{Funding Sources}

The authors declare that they did not receive any funding for this review.

\section{References}

1 Scottish Intercollegiate Guidelines Network (SIGN); 2010. Management of chronic venous leg ulcers. A national clinical guideline. Available from: www.sign.ac.uk/pdf/sign 120 . pdf.

2 Agale V. Chronic Leg Ulcers: Epidemiology, Aetiopathogenesis, and Management. Ulcers. 2013;2013:1-9.

3 Ruckley CV, Evans CJ, Allan PL, Lee AJ, Fowkes FG. Chronic venous insufficiency: clinical and duplex correlations. The Edinburgh Vein Study of venous disorders in the general population. J Vasc Surg. 2002 Sep; 36(3):520-5.

4 Abbade LP, Lastória S. Venous ulcer: epidemiology, physiopathology, diagnosis and treatment. Int J Dermatol. 2005 Jun;44(6): 449-56.

5 Valencia IC, Falabella A, Kirsner RS, Eaglstein WH. Chronic venous insufficiency and venous leg ulceration. J Am Acad Dermatol. 2001 Mar;44(3):401-21.
6 Barwell JR, Davies CE, Deacon J, Harvey K, Minor J, Sassano A, et al. Comparison of surgery and compression with compression alone in chronic venous ulceration (ESCHAR study): randomised controlled trial. Lancet. 2004 Jun;363(9424):1854-9.

7 Iglesias C, Nelson EA, Cullum NA, Torgerson DJ; VenUS Team. VenUS I: a randomised controlled trial of two types of bandage for treating venous leg ulcers. Health Technol Assess. 2004 Jul;8(29):iii.

8 Byrd L. CC Wound Identification: Differentiating Types of Ulcers: Arterial, Venous, Diabetic, and Pressure. Geriatr Nurs (Minneap). 2012;33(3):237-42.

9 Edwards H, Courtney M, Finlayson K, Lewis C, Lindsay E, Dumble J. Improved healing rates for chronic venous leg ulcers: pilot study results from a randomized controlled trial of a community nursing intervention. Int J Nurs Pract. 2005 Aug;11(4):169-76.

10 Ribu E, Haram R, Rustøen T. Observations of nurses' treatment of leg and foot ulcers in community health care. J Wound Ostomy Continence Nurs. 2003 Nov;30(6):342-50.
11 Templeton S, Telford K. Diagnosis and Management of Venous Leg Ulcers: A Nurse's Role? Wound Pract Res. 2010;18(2):72-9.

12 Guest JF, Ayoub N, McIlwraith T, Uchegbu I, Gerrish A, Weidlich D, et al. Health economic burden that different wound types impose on the UK's National Health Service. Int Wound J. 2017 Apr;14(2):322-30.

13 Graves N, Zheng H. Modelling the direct health care costs of chronic wounds in Australia. Wound Pract Res. 2014;22(1):20-33.

14 Ramstadius B. Leg ulcer management. Aust Nurs J. 1997 Aug;5(2):22.

15 Simka M, Majewski E. The social and economic burden of venous leg ulcers: focus on the role of micronized purified flavonoid fraction adjuvant therapy. Am J Clin Dermatol. 2003;4(8):573-81.

16 Vowden KR, Vowden P. The prevalence, management and outcome for patients with lower limb ulceration identified in a wound care survey within one English health care district. J Tissue Viability. 2009 Feb;18(1):139.

Asaf/Salim/Tuffaha 
17 Williams C. Rosidal K: a short-stretch compression bandage system. Br J Nurs. 2001 Mar;10(6):403-4.

18 Moffatt CJ, McCullagh L, O'Connor T, Doherty DC, Hourican C, Stevens J, et al. Randomized trial of four-layer and two-layer bandage systems in the management of chronic venous ulceration. Wound Repair Regen. 2003a May-Jun;11(3):166-71.

19 Partsch H, Damstra RJ, Tazelaar DJ, SchullerPetrovic S, Velders AJ, de Rooij MJ, et al. Multicentre, randomised controlled trial of fourlayer bandaging versus short-stretch bandaging in the treatment of venous leg ulcers. Vasa. 2001 May;30(2):108-13.

20 Wounds International. Consensus recommendations: simplifying venous leg ulcer management; 2015. Available from http:// www.3 mlearning.co.uk/media/1072/ 3m_14_4_consensus_web.pdf.

21 Parker CN, Finlayson KJ, Shuter P, Edwards HE. Risk factors for delayed healing in venous leg ulcers: a review of the literature. Int J Clin Pract. 2015 Sep;69(9):967-77.

22 Milic DJ, Zivic SS, Bogdanovic DC, Perisic ZD, Milosevic ZD, Jankovic RJ, et al. A randomized trial of the Tubulcus multilayer bandaging system in the treatment of extensive venous ulcers. J Vasc Surg. 2007 Oct;46(4): 750-5.

23 Polignano R, Bonadeo P, Gasbarro S, Allegra C. A randomised controlled study of four-layer compression versus Unna's Boot for venous ulcers. J Wound Care. 2004a Jan;13(1): 21-4.

24 Moffatt J, Simon A, Franks J, Connolly M, Fielden S, Groarke L, et al. Randomised Trial Comparing Two Four-Layer Bandage Systems in the Management of Chronic Leg Ulceration. Phlebology. 1999;14(4):139-42.

25 Vowden KR, Mason A, Wilkinson D, Vowden $\mathrm{P}$. Comparison of the healing rates and complications of three four-layer bandage regimens. J Wound Care. 2000 Jun;9(6):269-72.

26 Chalmers I, Hedges LV, Cooper H. A brief history of research synthesis. Eval Health Prof. 2002 Mar;25(1):12-37.
27 Ashby L, Gabe R, Ali S, Adderley U, Bland M, Cullum A, et al. Clinical and cost-effectiveness of compression hosiery versus compression bandages in treatment of venous leg ulcers (Venous leg Ulcer Study IV, VenUS IV): a randomised controlled trial. Lancet [Internet]. 2014 Mar;383(9920):871-879. Available from https://doi.org/10.1016/S01406736(13)62368-5

28 Finlayson KJ, Courtney MD, Gibb MA, O’Brien JA, Parker CN, Edwards HE. The effectiveness of a four-layer compression bandage system in comparison with Class 3 compression hosiery on healing and quality of life in patients with venous leg ulcers: a randomised controlled trial. Int Wound J. 2014 Feb;11(1):21-7.

29 Harding KG, Vanscheidt W, Partsch H, Caprini JA, Comerota AJ. Adaptive compression therapy for venous leg ulcers: a clinically effective, patient-centred approach. Int Wound J. 2016 Jun;13(3):317-25.

30 Harley J, Harcourt D, Hutchinson B, McLean $\mathrm{M}$, Long M. A comparative trial of long stretch compression bandaging versus multilayer compression bandaging in the treatment of chronic venous ulcers. Primary Intention. AWMA J. 2004;12(1):6-13.

31 Harrison MB, Vandenkerkhof EG, Hopman WM, Graham ID, Carley ME, Nelson EA; Canadian Bandaging Trial Group. The Canadian Bandaging Trial: evidence-informed leg ulcer care and the effectiveness of two compression technologies. BMC Nurs. 2011 Oct; 10(1):20-20.

32 Jünger M, Wollina U, Kohnen R, Rabe E. Efficacy and tolerability of an ulcer compression stocking for therapy of chronic venous ulcer compared with a below-knee compression bandage: results from a prospective, randomized, multicentre trial. Curr Med Res Opin. 2004 Oct;20(10):1613-23.

33 de Abreu AM, de Oliveira BG. A study of the Unna Boot compared with the elastic bandage in venous ulcers: a randomized clinical trial [RLAE]. Rev Lat Am Enfermagem. 2015 JulAug;23(4):571-7.

34 Meyer FJ, McGuinness CL, Lagattolla NR, Eastham D, Burnand KG. Randomized clinical trial of three-layer paste and four-layer bandages for venous leg ulcers. Br J Surg. 2003 Aug;90(8):934-40.
35 Nelson EA, Iglesias CP, Cullum N, Torgerson DJ; VenUS I collaborators. Randomized clinical trial of four-layer and short-stretch compression bandages for venous leg ulcers (VenUS I). Br J Surg. 2004 Oct;91(10):1292-9.

36 Polignano R, Guarnera G, Bonadeo P. Evaluation of SurePress Comfort: a new compression system for the management of venous leg ulcers. J Wound Care. 2004b Oct;13(9):38791.

37 Escaleira R, Cardoso M, Rego J, Macedo P, Midões A. Efficacy of a two-component compression system for the therapy of venous leg ulcers. J Wound Care. 2010 Mar; 19(3):104-9.

38 Phillips TJ, Machado F, Trout R, Porter J, Olin J, Falanga V. Prognostic indicators in venous ulcers. J Am Acad Dermatol. 2000 Oct;43(4): 627-30.

39 Keller A, Müller ML, Calow T, Kern IK, Schumann H. Bandage pressure measurement and training: simple interventions to improve efficacy in compression bandaging. Int Wound J. 2009 Oct;6(5):324-30.

40 Lee AJ, Dale JJ, Ruckley CV, Gibson B, Prescott RJ, Brown D. Compression therapy: effects of posture and application techniques on initial pressures delivered by bandages of different physical properties. Eur J Vasc Endovasc Surg. 2006 May;31(5):542-52.

41 Partsch H, Clark M, Mosti G, Steinlechner E, Schuren J, Abel M, et al. Classification of compression bandages: practical aspects. Dermatol Surg. 2008 May;34(5):600-9.

42 Benigni JP, Lazareth I, Parpex P, Gerard JL, Alves M, Vin F, et al. Efficacy, safety and acceptability of a new two-layer bandage system for venous leg ulcers. J Wound Care. 2007 Oct;16(9):385-90.

43 Dolibog P, Franek A, Taradaj J, Dolibog P, Blaszczak E, Polak A, et al. A comparative clinical study on five types of compression therapy in patients with venous leg ulcers. Int J Med Sci. 2013 Dec;11(1):34-43.

44 Szewczyk MT, Jawień A, Cierzniakowska K, Cwajda-Białasik J, Mościcka P. Comparison of the effectiveness of compression stockings and layer compression systems in venous ulceration treatment. Arch Med Sci. 2010 Oct; 6(5):793-9. 